PROCEEDINGS OF THE

AMERICAN MATHEMATICAL SOCIETY

Volume 126, Number 12, December 1998, Pages 3743-3747

S 0002-9939(98)04509-2

\title{
ON COMPLETE METRIC SPACES CONTAINING THE SIERPIŃSKI CURVE
}

\author{
JANUSZ R. PRAJS
}

(Communicated by Alan Dow)

\begin{abstract}
It is proved that a complete metric space topologically contains the Sierpiński universal plane curve if and only if it has a subset with so-called bypass property, i.e. it has a subset $K$ containing an arc such that for each $a \in K$ and for each open arc $A \subset K$ with $a \in A$, there exists an arbitrary small arc in $K \backslash\{a\}$ joining the two components of $A \backslash\{a\}$.
\end{abstract}

Investigating homogeneous continua which contain an arc, a characterization of complete metric spaces that topologically contain the Sierpiński universal plane curve has been obtained (Theorem 1 below). To formulate this result we need the following definition.

Definition 1. A metric space $X$ has the bypass property if

(1) $X$ contains an arc, and

(2) for every arc $x y$ in $X$ and every point $z \in x y \backslash\{x, y\}$ and $\varepsilon>0$ there is an arc $x^{\prime} y^{\prime}$ in $X \backslash\{z\}$ with diameter $<\varepsilon$ joining the two components of $x y \backslash\{z\}$.

Theorem 1. A complete metric space $X$ topologically contains the Sierpinski universal plane curve if and only if $X$ contains a subset with the bypass property.

The aim of this note is to prove this theorem. Observe that sets with the bypass property need not be 'nice'. They may be of the first category in themselves and they may have degenerate components or arc components. They may be 'poor', i.e. composed of countably many arcs in each boundary in the set. The theorem is a tool to conclude that complete metric spaces containing such a 'poor' subset are 'rich' in some sense - they topologically contain all planar one-dimensional spaces.

The theorem is related to the results of [3].

The paper is ended with a corollary on homogeneous continua and with three related questions.

To prove Theorem 1 we need the following lemma.

Lemma 2. Let $X$ have the bypass property. For every connected graph $G \subset X$ without end points and for every $\varepsilon>0$ there is a connected graph $H \subset X$ without end points such that

Received by the editors December 19, 1996 and, in revised form, April 21, 1997.

1991 Mathematics Subject Classification. Primary 54F15, 54F65, 54F50, 54C25.

Key words and phrases. Bypass property, embedding, homogeneity, local separating point, Sierpiński curve.

The author expresses grateful thanks to Prof. K. Omiljanowski for his help in the preparation of this paper.

(C)1998 American Mathematical Society 
(a) $H$ is the union of $G$ and a finite number of arcs of diameter $<\varepsilon$ each intersecting $G$, and

(b) for any arc $x y \subset G$ and any point $z \in x y \backslash\{x, y\}$ there is an arc in $H \backslash\{z\}$ joining $x, y$ and contained in the $\varepsilon$-neighborhood of $x y$.

Proof. By the bypass property of $X$ for each $\varepsilon$-small arc $L$ in $G$ and for each of its points $z$ (except its end points) there is an $\varepsilon$-small arc $A_{L, z}$ in $X \backslash\{z\}$ joining the two components of $L \backslash\{z\}$. (Of course we can assume that no vertex of $G$ belongs to $A_{L, z}$.) Let $U_{L, z}$ stand for an open arc satisfying $z \in U_{L, z} \subset \overline{U_{L, z}} \subset L \backslash A_{L, z}$. Observe that $A_{L, z}$ joins the two components of $L \backslash\left\{z^{\prime}\right\}$ for any $z^{\prime} \in U_{L, z}$. We will say that the $\operatorname{arc} A_{L, z}$ corresponds to $U_{L, z}$.

Of course $U_{L, z}$ is a neighborhood of $z$ in $L$, and also in $G$ if $z$ is not a vertex of G.

For each vertex $v$ of $G$ fix a finite collection $\left\{U_{L_{1}, v}, U_{L_{2}, v}, \ldots, U_{L_{i_{v}}, v}\right\}$ defined as above such that each pair of adjacent edges at $v$ is joined by some $U_{L_{k}, v}$.

The set $G \backslash \bigcup\left\{\bigcup_{k=1}^{i_{v}} U_{L_{k}, v}: v\right.$ is a vertex of $\left.G\right\}$ contains no vertex and it is compact, hence there is a finite cover $\left\{U_{L_{z_{1}}, z_{1}}, U_{L_{z_{2}}, z_{2}}, \ldots, U_{L_{z_{m}}, z_{m}}\right\}$ of it.

Let (for simplicity) $U_{1}, U_{2}, \ldots, U_{n}$ denote all these sets of this cover and all the above mentioned sets $U_{L_{k}, v}$ for all vertices $v$ in $G$. Denote by $A_{1}, A_{2}, \ldots, A_{n}$ the arcs of the form $A_{L, z}$ which correspond to sets $U_{1}, U_{2}, \ldots, U_{n}$.

Observe that the union $H=G \cup A_{1} \cup A_{2} \cup \ldots \cup A_{n}$ has all the desired properties except one; this union may not be a graph.

If it is the case, it may be improved inductively. Suppose that $G \cup A_{1} \cup A_{2} \cup \ldots \cup A_{i}$ is a graph but $G \cup A_{1} \cup A_{2} \cup \ldots \cup A_{i} \cup A_{i+1}$ is not a graph. Then the subset $A_{i+1} \backslash$ $\left(G \cup A_{1} \cup A_{2} \cup \ldots \cup A_{i}\right)$ of the arc $A_{i+1}$ has infinitely many components. Almost all of them may be replaced by finitely many small arcs in $G \cup A_{1} \cup A_{2} \cup \ldots \cup A_{i}$ so that some arc $A_{i+1}^{\prime}$ close to $A_{i+1}$ is obtained satisfying:

$A_{i+1}^{\prime}$ corresponds to the same set $U_{i+1}$ as $A_{i+1}$, and

$A_{i+1}^{\prime} \backslash\left(G \cup A_{1} \cup A_{2} \cup \ldots \cup A_{i}\right)$ has finitely many components.

Hence $G \cup A_{1} \cup A_{2} \cup \ldots \cup A_{i} \cup A_{i+1}^{\prime}$ is a graph.

For any metric space $X$, any set $Y \subset X$ and for any $\varepsilon>0$ define

$$
T_{\varepsilon}(Y, X)=\bigcup\{A: A \text { is an } \operatorname{arc} \operatorname{in} X, \operatorname{diam} A<\varepsilon \text { and } A \cap Y \neq \emptyset\} .
$$

Observe the following easy property of the operator $T$ :

$$
T_{\alpha}\left(T_{\beta}(Y, X), X\right) \subset T_{\alpha+\beta}(Y, X)
$$

for all positive numbers $\alpha, \beta$.

Construction of $G_{n}$ 's. Fix any metric space $B$ with the bypass property. Take any simple closed curve $G_{1}$ in $B$ and put $\varepsilon_{0}=1$. We inductively construct an increasing sequence $G_{n}$ of graphs in $B$ and a sequence $\varepsilon_{n}$ of positive numbers as follows.

Assume $G_{1}, \ldots, G_{n}$ and $\varepsilon_{0}, \ldots, \varepsilon_{n-1}$ are already constructed. Let

$$
\varepsilon_{n}<\min \left\{\varepsilon_{n-1} / 2 ; 1 /(8 n)\right\}
$$

be a fixed positive number such that for any two points $x, y \in G_{n}$ satisfying $d(x, y)<5 \varepsilon_{n}$ there exists an arc $A \subset G_{n}$ with $x, y \in A$ and $\operatorname{diam} A<1 /(3 n)$. 
Let $G_{n+1}$ be the graph $H$ guaranteed by Lemma 2 for graph $G=G_{n}$ and for $\varepsilon=\varepsilon_{n}$. Let $F=\bigcup_{n \in \mathcal{N}} G_{n}$.

The above construction plays a crucial role in the proof of the main result of the paper. Now, we come to prove some of its properties.

First, notice that $G_{n+1}=T_{\varepsilon_{n}}\left(G_{n}, G_{n+1}\right) \subset T_{\varepsilon_{n}}\left(G_{n}, F\right)$ by the construction of $G_{n}$ 's (see Lemma 2(a)). Applying (1), by the induction we obtain

$$
G_{n+k}=T_{2 \varepsilon_{n}}\left(G_{n}, G_{n+k}\right) \subset T_{2 \varepsilon_{n}}\left(G_{n}, F\right)
$$

for each pair of positive integers $n, k$.

Property 3. For any $\varepsilon>0$ there exists a $\delta>0$ such that for each triple of points $p, x, y \in F$ satisfying $x \neq p \neq y$ and $d(x, y)<\delta$, there exists an arc $A \subset F \backslash\{p\}$ with $x, y \in A$ and $\operatorname{diam} A<\varepsilon$.

Proof. Given any $\varepsilon>0$, take a positive integer $n$ such that $1 / n<\varepsilon / 2$, and put $\delta=\varepsilon_{n}$. Let points $p, x, y \in F$ satisfy $x \neq p \neq y$ and $d(x, y)<\delta$. Then there is a $k \in \mathcal{N}$ such that $x, y \in G_{n+k}$. By (2) there are points $a_{x}, a_{y} \in G_{n}$ and arcs $A_{x}, A_{y}$ in $G_{n+k}$ such that $x, a_{x} \in A_{x} ; y, a_{y} \in A_{y}$ and $\operatorname{diam} A_{x}, \operatorname{diam} A_{y}<2 \varepsilon_{n}$. We have $d\left(a_{x}, a_{y}\right)<\operatorname{diam} A_{x}+\delta+\operatorname{diam} A_{y}<5 \varepsilon_{n}$, thus, by the definition of $\varepsilon_{n}$, there is an $\operatorname{arc} A_{1} \subset G_{n}$ such that $a_{x}, a_{y} \in A_{1}$ and $\operatorname{diam} A_{1}<1 /(3 n)$. Since $\operatorname{diam} A_{x}, \operatorname{diam} A_{y}<2 \varepsilon_{n}<1 /(4 n)$, then the diameter of the set $K=A_{x} \cup A_{1} \cup A_{y}$ is $<1 / n<\varepsilon / 2$. Next, $K$ is a locally connected continuum in $G_{n+k}$, thus it contains an $\operatorname{arc} A$ in $G_{n+k}$ with $x, y \in A$. If $p \in A$, we apply the construction of $G_{i}$ 's and condition (b) of Lemma 2, to modify arc $A$ and obtain an arc $A^{\prime}$ in $G_{n+k+1}$ such that $x, y \in A^{\prime} \subset G_{n+k+1} \backslash\{p\}$ and $\operatorname{diam} A^{\prime}<2 \cdot 1 / n<\varepsilon$.

Recall that a metric space $X$ is said to have property $S$ provided for any $\varepsilon>0$, space $X$ may be represented as the finite union of its connected subsets each having diameter less than $\varepsilon$ (see [5], Chapter I, §15).

Property 4. Set $F$ has property $S$.

Indeed, let $n$ satisfy $5 \varepsilon_{n}<\varepsilon$. Since $G_{n}$ is a graph, it can be represented as the finite union of $\operatorname{arcs} A_{1}, \ldots, A_{k}$ each having diameter $<\varepsilon_{n}$. Hence $F=T_{2 \varepsilon_{n}}\left(G_{n}, F\right)=$ $T_{2 \varepsilon_{n}}\left(A_{1}, F\right) \cup \ldots \cup T_{2 \varepsilon_{n}}\left(A_{k}, F\right)$ by $(2)$. The reader can easily observe that the above union is that required.

Theorem 5. If a complete metric space $X$ contains a subspace $B$ with the bypass property, then $X$ contains a locally connected curve with no local separating point.

Proof. Assumming some extra condition, we repeat the construction of graphs $G_{1}, G_{2}, \ldots$ in $B$ (see the Construction of $G_{n}$ 's just below (1)), to obtain the closure of their union having the desired properties. In this construction we employ the fact that $G_{n}$ 's are compact $A N R$ 's.

The modification is the following. On the initial step of the construction we take a neighborhood $V_{1}$ of $G_{1}$ in $X$, admitting a 1-retraction $r_{1}: V_{1} \rightarrow G_{1}$ (i.e. a retraction satisfying diam $r_{1}^{-1}(y)<1$ for each $\left.y \in G_{1}\right)$.

On each inductive step we take $\varepsilon_{n}$ additionally so small, that $N_{\varepsilon_{n}}\left(G_{n}\right) \subset V_{n}$. (The previous restrictions on $\varepsilon_{n}$ are still satisfied.) Finally, we take any neighborhood $V_{n+1} \subset V_{n}$ of $G_{n+1}$ in $X$, admitting an $\varepsilon_{n}$-retraction $r_{n+1}: V_{n+1} \rightarrow G_{n+1}$. The construction is complete.

Put $Z=\bar{F}$, where $F=\bigcup_{n \in \mathcal{N}} G_{n}$ and the closure is taken in $X$. 
Of course $Z$ is a closed connected set. Notice that $Z \subset \bigcap_{n \in \mathcal{N}} V_{n}$. Thus for each $n$ set $Z$ has $\varepsilon_{n-1}$-retraction $r_{n} \mid Z$ onto graph $G_{n}$. Therefore, $Z$ is one-dimensional.

By the construction $F$ satisfies the conclusions of Properties 3 and 4.

Since $F$ has property $S$ by Property 4, its closure $Z$ does also (see [5], Chapter I, $\S 15,(15.3)$, p.20). Thus $Z$ is locally connected. Moreover, again by property $S$, set $Z$ is a totally bounded, complete metric subspace of $X$. Hence $Z$ is compact.

One can easily verify that Property 3 for $F$ implies that $Z$ has no local separating point.

Proof of Theorem 1. By Theorem 5 space $X$ contains a locally connected curve $Z$ with no local separating point. If some open (in $Z$ ) subset of $Z$ is embeddable in the plane, then this subset is homeomorphic to some open subset of the Sierpiński universal plane curve (see [6]). Otherwise $Z$ is the Menger universal curve (see [1], [2] or [4], Th.4.11). In both cases $Z$ contains (topologically) a copy of the Sierpiński universal plane curve.

The converse implication is obvious.

To show some applications of the result let us state the following definition.

Definition 2. A point $p$ of a space $X$ is said to be a local arc separating point in $X$ provided there exists a neighborhood $U$ of $p$ in $X$ and an arc $A \subset U$ such that $A \backslash\{p\}$ meets two different arc components of $U \backslash\{p\}$.

Observe that any space with the bypass property contains no local arc separating point.

By Theorem 1 and by the universal properties of the Sierpiński curve we obtain the following corollary on homogeneous continua.

Corollary 6. For any homogeneous continuum $X$ containing an arc either $X$ topologically contains all planar one-dimensional sets, or each point of $X$ is a local arc separating point in $X$.

We end the paper with three questions connected with the bypass property and homogeneous continua. In these questions $X$ denotes an arbitrary homogeneous continuum.

Question 1. If X contains arbitrarily small simple closed curves, does it topologically contain the Sierpiński curve?

Question 2. If $X$ contains the Sierpinski curve, does $X$ have the bypass property? (Equivalently, does $X$ have no local arc separating point?)

Question 3. If $X$ contains the Sierpinski curve, does $X$ contain either two-dimensional disc, or the Menger universal curve?

\section{REFERENCES}

[1] R. D. Anderson, A characterization of the universal curve and the proof of its homogeneity, Ann. of Math. 67 (1958), 313-324. MR 20:2675

[2] R. D. Anderson, One-dimensional continuous curves and a homogeneity theorem, Ann. of Math. 68 (1958), 1-16. MR 20:2676

[3] P. Krupski and H. Patkowska, Menger curves in Peano continua, Colloq. Math. 70 (1996), 79-86. CMP 96:08

[4] J C. Mayer, L. G. Oversteegen and E. D. Tymchatyn, The Menger curve characterization and extension of homeomorphisms of non-locally-separating closed subsets, Dissert. Math. 252 (1986), 1-45. MR 87m:54106 
[5] G. T. Whyburn, Analytic topology, Amer. Math. Soc. Colloq. Publ., vol.28, Amer. Math. Soc., Providence, R. I., 1942. MR 4:86b

[6] G. T. Whyburn, Topological characterization of the Sierpinssi curve, Fund. Math. 45 (1958), 320-324. MR 20:6077

Institute of Mathematics, Opole University, ul. Oleska 48, 45-052 Opole, Poland

E-mail address: jrprajs@math.uni.opole.pl 